\title{
JOURNAL.RU
}

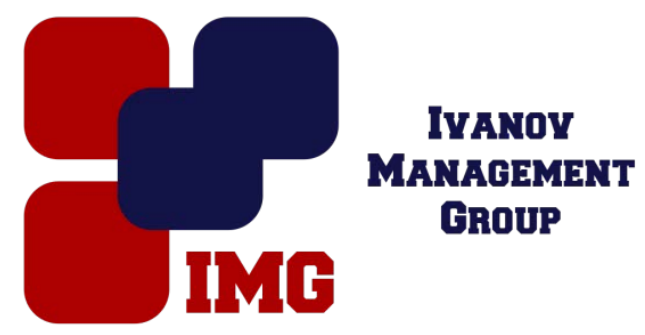

Кучеров А.С., Борзов Д.Б. Юго-Западный государственный университет Курск, Россия

doi: 10.18411/lj-28-02-2017-1-06

idsp 000001:lj-28-02-2017-1-06

\section{Планирование размещения программ в динамических мультипроцессорных системах}

В настоящее время многопроцессорная техника находит все большее применение в различных сферах жизни[1]. При этом часто используются сетевые многопроцессорные системы (СМC)[2].

Идея СМС основана на взаимодействии отдельных процессорных модулей по беспроводной связи с использованием соответствующих стандартов высокоскоростной передачи данных, т.к. LTE (LongTermEvolution), Wi-Fi (WirelessFidelity) и т.п.

Главная проблема СМС - оптимальное распределение задач среди входящих в систему вычислительных устройств. Задача становится сложнее, если положение отдельных ВМ в пространстве меняется во времени. В таких случаях используется модель локальной вычислительной сети.

Локальная вычислительная сеть (ЛВС) - это совокупность компьютеров и компьютерного оборудования (коммутаторы, маршрутизаторы, сервера), находящихся, как правило, на территории какого-либо предприятия/организации, соединенных между собой проводными или беспроводными каналами связи и выполняющих определенную вычислительно емкую задачу.

Для достаточно быстрой работы ЛВС необходимы надежные и быстрые каналы связи между ЭВМ, входящими в нее. Все каналы связи локальной вычислительной сети описываются ее топологией.

Топология сети представляет собой взвешенный ориентированный граф (каналы приема и передачи могут иметь разную пропускную способность.) Для наглядного представления особенностей сети предлагается рассматривать матрицу расстояний - квадратную матрицу, значение ячейки которой равно величине, обратной пропускной способности канала между i-й и j-й вычислительной машиной.

$$
R=\left[\begin{array}{ccc}
r_{11} & \cdots & r_{1 n} \\
\vdots & \ddots & \vdots \\
r_{n 1} & \cdots & r_{n n}
\end{array}\right],
$$

где rij- расстояние между і-й и ј-йВМ в СМС. 
Если гіјблизко к 0, то пропускная способность канала высокая. Соответственно, данные от і-й к ј-й вычислительной машине будут передаваться без больших задержек. Если гіјблизко к бесконечности, то пропускная способность канала низкая; следовательно, желательно избегать прямого обмена большими порциями данных между этими двумя машинами.

В проектируемой модели каждая вычислительная машина может выполнять следующие роли:

1. Рабочая станция - BM, непосредственно занятая частью общей вычислительной задачи;

2. Сервер - ВМ, распределяющая задачи между рабочими станциями, осуществляющая информационный обмен и хранящая вычислительную информацию;

3. Резервное хранилище - ВМ, хранящая вычислительную информацию.

Локальная вычислительная сеть может включать в себя один сервер, несколько резервных хранилищ и рабочих станций. ВМ, исполняющая роль сервера или резервного хранилища, может также участвовать в общих вычислениях, то есть являться одновременно рабочей станцией.

Преимуществом разрабатываемой модели перед имеющимися является ее устойчивость к следующим факторам:

- выход из строя рабочей станции или ееотключение от сети: опрос рабочей станции производится по тайм-ауту, определяемому в зависимости от вычислительной сложности задачи;

- выход из строя сервера: в этом случае все вычислительные данные могут быть получены из резервных хранилищ;

- изменение топологии сети: изменение топологии влечет за собой изменение матрицы расстояний (1), и, как следствие, перераспределение задач между рабочими станциями ЛВС.

\section{Литература}

1. Борзов Д.Б., Титов В.С. Вопросы проектирования и динамической реконфигурации топологии систем логического управления в системах высокой готовности: монография / Юго-Зап. гос. ун-т. -Курск, 2015. -282 с.

2. Данильченко Н.В., Макеев С.Н. Масштабируемый мультипроцессор для цифровой обработки сигналов // Проблемы разработки перспективных микро- и наноэлектронных систем - 2012: сб. тр. /под общ. ред. акад. РАН А.Л.Стемпковского. - М.: ИППМ РАН, 2012. - С. 495-500. 